\title{
CRITERIOS QUE UTILIZAN LOS ALUMNOS UNIVERSITARIOS DE PRIMER CICLO PARA DEFINIR SER VIVO
}

\author{
MONDELO ALONSO, MATILDE, MARTÍNEZ LOSADA, C. y GARCÍA BARROS, SUSANA \\ Facultade de Ciencias da Educación. Universidade da Coruña. Paseo de Ronda 47. 15011 A Coruña.
}

\section{SUMMARY}

In our work we have tried to find out to what extent university students in their first three courses apply those criteria defining «the living things» concept. We have verified that they focus their attention on physiological aspects especially perceptible and macroscopic ones rather than on universal and microscopic characteristics.

\section{INTRODUCCIÓN}

La presencia de contenidos relativos a los seres vivos en general, y al ser humano en particular, es constante en los currículos de la educación formal. Su tratamiento gradual y continuado a lo largo de la enseñanza, indicativo de la amplitud y complejidad de la materia que nos ocupa, debería posibilitar que los alumnos lograsen adquirir en último término un concepto de vida acorde con la visión científica. El logro del mencionado nivel conceptual requiere: aprehender el concepto de ser vivo y reconocer, por tanto, que los seres que tienen o han tenido vida poseen unas características propias que los diferencian de la materia inerte; conocer que la vida adopta diversidad de formas y que éstas presentan distintos niveles de complejidad estructural; iniciarse en el estudio de la célula como unidad anatómico-funcional, de sus estructuras y procesos metabólicos y asumir, finalmente, que tras la diversidad de seres vivientes, subyace la uniformidad estructural y funcional.

En este sentido, la investigación en didáctica de las ciencias ha puesto de manifiesto que los alumnos tienen dificultades para apropiarse de conocimientos fundamentales respecto a distintos procesos vitales como respiración (Haslam y Treagust, 1987; Anderson et al., 1990; Banet y Núñez, 1990; Songer y Mintzes, 1994), fotosíntesis (Arnold y Simpson, 1980; Haslam y Treagust, 1987; Anderson et al., 1990), herencia (revisión de Wood-Robinson, 1994), etc. Sin embargo, las investigaciones relativas al concepto de vida, especialmente en niveles no elementales, son más escasas. Desde el clásico estudio de Piaget (1929) sobre la evolución del concepto de ser vivo en los niños, se realizaron otros trabajos dirigidos a conocer las características que estudiantes de distintas edades atribuyen a los organismos. El movimiento ha sido uno de los atributos ampliamente asociados con lo vivo (Piaget, 1929; Russell y Dennis, 1939; Lucas et al., 1979). Asimismo, se ha detectado que incluso niños de corta edad ( 8 años) asignan, tanto a los seres vivos como a los inertes, otras características como alimentación, respiración, reproducción (Looft, 1974) y crecimiento, muerte, sensaciones, etc. (Smeets, 1973). La utilización de criterios diversos para definir lo 
vivo ha sido plenamente corroborada por Lucas y otros (1979) al apreciar que un considerable porcentaje de alumnos de distintos niveles educativos (8-16 años) sugerían criterios basados en estructuras externas, incrementándose con el nivel las referencias a estructuras internas así como a funciones fisiológicas y comportamentales. En la misma línea se sitúan los resultados obtenidos por Osaki y Samiroden (1990) en niños de 9, 11 y 13 años de edad, aunque estos autores detectan un predominio de las características fisiológicas y del comportamiento frente a las morfológicas. Por otra parte, Brumby (1982), al enfrentar a estudiantes universitarios de biología (18-22 años) con problemas relativos al concepto de vida, observa que el movimiento y la textura (duro, blando, etc.) son los criterios más utilizados para determinar si un ser está vivo, aunque también hacen referencia a otras características «tradicionales» como respiración externa, crecimiento, reproducción, nutrición, irritabilidad y excreción. La citada autora indica también que, si bien los alumnos se basan en la presencia de células y en la composición química orgánica para diferenciar al ser vivo de la materia inerte, apenas mencionan moléculas concretas como el DNA, ni se refieren a su replicación o a transformaciones energéticas celulares.

Sin embargo, el hecho de que los estudiantes atribuyan diversas características a los seres vivos no significa que reconozcan las propiedades básicas y comunes a todos ellos. Por el contrario, el concepto de vida que poseen suele variar de unos organismos a otros, lo que denota un conocimiento ligado al contexto (Tamir et al., 1981; Carey, 1985; Stavy y Wax, 1989; Bell y Freyberg, 1991; Strommen, 1995).

El principal objetivo que se pretende en este trabajo es determinar en qué medida estudiantes universitarios de distintas especialidades asumen, dentro de la diversidad de los seres vivos, la uniformidad de estructura y función. Se trata, en definitiva, de conocer qué criterios utilizan para diferenciar ser vivo de materia inerte. Como objetivo secundario se pretende averiguar qué procedimientos científicos utilizan para avalar sus planteamientos teóricos.

\section{HIPÓTESIS}

Basándonos tanto en los resultados obtenidos en diversos estudios sobre el tema que nos ocupa como en nuestra propia experiencia docente, suponíamos que los estudiantes utilizarían fundamentalmente como soporte conceptual la definición de ser vivo más generalizada en el alumnado («nace», «crece»...) las características vitales relativas a procesos fisiológicos (respiración, reproducción, alimentación, movimiento...) o los aspectos anatómicos especialmente perceptibles macroscópicamente (sistemas, órganos), mientras que la estructura celular, la presencia de moléculas específicas (ADN, proteínas, etc.) o los procesos metabólicos serían tenidos en cuenta en menor medida.
Por otra parte, se preveía que los estudiantes tuvieran dificultades para aplicar procedimientos científicos tales como la propuesta de hipótesis o el diseño de experiencias para contrastarlas.

\section{METODOLOGÍA}

En este estudio han sido consultados un total de 226 estudiantes universitarios: 42 de biológicas $\left(1^{\circ}\right.$ y $2^{\circ}$ cursos) y 184 de distintas especialidades de Magisterio $\left(1^{\circ}\right.$ de educación primaria y $1^{\circ}$ de educación infantil $)$.

Para recabar la información de los alumnos se elaboró un cuestionario similar a otros ya ensayados en estudios sobre las características que los alumnos atribuyen al «ser vivo» en contraposición a la materia inerte (Lucas et al., 1979; Brumby, 1981; Osaki y Samiroden, 1990). El cuestionario consta de una única pregunta de libre respuesta que debe aplicarse a las representaciones de tres seres vivos muy conocidos (un niño, un árbol y una vaca) y a la de un objeto inerte (un cristal de aragonito no identificable como tal en la copia).

Se ha incluido la representación de un niño con objeto de conocer si los alumnos atribuyen a la especie humana características semejantes a las de otros seres vivos o si, por el contrario, se decantan por la utilización de criterios específicos, máxime cuando se ha detectado que no siempre la identifican como un «animal» (Bell y Freyberg, 1991). Por tal motivo, y con el fin de realizar comparaciones, también se seleccionó otro animal muy común -vaca-. Además, era preciso incluir un vegetal, ya que muchos estudiantes los consideran «menos vivos» que a los animales (Caballer y Giménez, 1992; Mondelo et al., 1994). Por otra parte, parecía interesante proponer un ejemplar desconocido para los alumnos, en la línea seguida por los autores ya mencionados (Lucas et al., 1979; Brumby, 1981; Osaki y Samiroden, 1990). Para ello, elegimos la copia de una lámina de aragonito porque ofrecía un aspecto ambiguo, que tanto se podría asociar con un conjunto de estalagmitas como con una colonia de corales.

El enunciado de la pregunta es el siguiente: Los seres vivos, en cualquier forma que se presenten, poseen unas características que nos permiten diferenciarlos claramente de la materia inerte. ¿Qué experimentos se podrían realizar para saber si los siguientes ejemplares son seres vivos?

Como se puede apreciar, en la cuestión se especifica que los seres vivos y los inertes presentan diferencias esenciales. Así, la ciencia actual defiende el principio de universalidad en el sentido de que todos los organismos comparten los mismos tipos de moléculas hereditarias, equivalentes mecanismos de transmisión, recombinación, expresión y variación, así como el código del que depende su expresión (Jouve, 1996). En este estudio, sin embargo, hemos dado por válida la concepción de los seres vivos como sistemas materiales altamente organizados, con una constitución básica celular dotada de 
vida propia (Mondelo et al., 1994) y que presentan diversos niveles de organización.

En la pregunta también se solicita la propuesta de experimentos con el fin de diferenciar lo vivo de lo inerte. Si bien un experimento se debe entender como «aquella clase de experiencia científica en la cual se provoca deliberadamente algún cambio y se observa e interpreta su resultado con alguna finalidad cognitiva» (Bunge, 1969), en este trabajo se utiliza en un sentido amplio, abarcando un proceso que implica la emisión de hipótesis y su contrastación a través de la observación o la experimentación. En cualquier caso, se introdujo el término experimento porque los sondeos preliminares a este trabajo mostraron que, mediante otro tipo de preguntas más genéricas, los alumnos se limitaban a proponer descripciones macroscópicas obvias.

Los alumnos contestaron a la pregunta formulada en su clase habitual de biología o de didáctica de la biología. En el momento de su presentación se les indicó que debían proponer todos los experimentos que considerasen oportunos y que, siempre según su criterio, éstos podían ser, o no, los mismos para los cuatro ejemplos presentados.

Para la categorización de las respuestas se ha analizado una muestra aleatoria de las respuestas obtenidas -un $10 \%$ de las mismas, aproximadamente-. Al detectarse un elevado número de observaciones y descripciones, se estableció una categoría específica en este sentido. Así, con relación a los procedimientos propuestos, todas las respuestas se clasificaron en dos grupos: a) las que proponían simplemente observaciones o descripciones; b) las que planteaban algún tipo de experimento.

Independientemente de los procedimientos científicos propuestos, todas las respuestas se categorizaron atendiendo al marco conceptual en el que se fundamentaban, clasificándose en dos grupos: $a$ ) respuestas basadas en criterios fisiológicos -alimentación, nutrición, reproducción, etc.-, considerando conjuntamente las referencias a alimentación y nutrición con el fin de respetar la terminología usada por el alumnado; y $b$ ) respuestas basadas en aspectos estructurales tanto macroscópicos como microscópicos -sistemas, órganos, célula, composición química-. Todo el proceso ha sido realizado por dos miembros del equipo, tomando una decisión consensuada en caso de discrepancia.

\section{RESULTADOS}

En primer lugar debemos señalar que los estudiantes respondieron ampliamente a la cuestión planteada, dando varias respuestas para cada uno de los ejemplos presentados. Se ha obtenido un total de 3.179 respuestas y el número medio de las mismas por alumno y objeto varía ligeramente en función tanto de los colectivos consultados como del propio objeto (ser vivo o inerte) considerado. El número medio de respuestas por alumno fue superior, para todos los ejemplares, en el grupo de magisterio que en el de biológicas, recayendo la media más alta en el ejemplar «niño» $(4,7$ y 4,2 respectivamente) y la más baja en «cristal» $(3,2$ y 2,0$)$. En segundo lugar, conviene precisar que el porcentaje de alumnos que no varió sus respuestas, es decir, que utilizó siempre los mismos criterios en los cuatro ejemplos, es inferior al $5 \%$.

\section{Procedimientos propuestos}

La gran mayoría de las respuestas obtenidas son de tipo observacional o descriptivo, siendo minoritarias las que presentan experimentos en el amplio sentido del término. En concreto, las observaciones directas superan en todos los ejemplares el 61,5\% del total de respuestas emitidas. Las propuestas experimentales se aplicaron en mayor medida a «árbol», en el grupo de biológicas, y a «cristal» en el de magisterio, observándose un ligero incremento de las mismas en este último colectivo.

Un análisis cualitativo de las respuestas observacionales o simplemente descriptivas, indica que las expresiones más utilizadas por los alumnos para comprobar si un ejemplar concreto pertenece o no al mundo de los seres vivos, fueron: observar..., ver si..., mirar..., los seres vivos..., saber si..., etc., todos ellos tendentes a poner de manifiesto la existencia de características vitales tales como nacimiento, muerte, nutrición, reproducción, presencia de estructuras, etc. o la necesidad de alimentos, agua, oxígeno o luz. Algunas frases textuales fueron: «observar si respira, se mueve, se nutre, observar si se reproduce, los seres vivos nacen, crecen, se relacionan, se reproducen y mueren, ver si tiene pulso, si le late el corazón, si tiene sangre, los seres vivos también se mueren y se descomponen, miro si el árbol necesita agua y oxígeno y luz solar para vivir, hacer un análisis interno, observando órganos, sistemas de órganos y su funcionamiento, ver si está constituido por células, analizar su composición química, si tiene moléculas orgánicas como ácidos nucleicos, lípidos, proteínas».

Con relación a los experimentos propuestos debemos indicar que en general los alumnos se limitaron a enunciar la «acción» concreta que podría realizarse sobre el ejemplar -darle o privarle de alimento, pincharlo, etc.y, en ocasiones, a señalar la consecuencia de la misma -comería, se muere, chillaría-. En la tabla I se recogen ejemplos de pruebas representativas. Como puede observarse, en ningún caso se explicita la hipótesis de partida aunque a veces ésta aparece como resultado esperado. Tampoco está presente la técnica, ni siquiera rudimentaria, que debería seguirse para la toma de datos, análisis de éstos y obtención de conclusiones. Por otra parte, no siempre se sugiere el resultado esperado. En este sentido, debemos señalar que se aprecian diferencias entre los dos grupos de alumnos. Concretamente, en la mayoría de los experimentos propuestos por los alumnos de magisterio se omite dicho resultado, mientras que en los de biológicas se especifica mayoritariamente. 
Tabla I

Algunos experimentos propuestos por los alumnos.

\begin{tabular}{|c|c|c|}
\hline EJEMPLAR & EXPERIMENTO & RESULTADO ESPERADO \\
\hline NIÑO & $\begin{array}{l}\text { Darle alimentos } \longrightarrow \\
\text { Poner un cristal delante de la boca } \longrightarrow \\
\text { Pincharlo con un alfiler } \\
\text { Comprobar que se mueve } \\
\text { Tomarle el pulso } \longrightarrow \\
\text { Tomar una muestra de tejidos } \longrightarrow\end{array}$ & $\begin{array}{l}\text { Si fuera un ser vivo comería } \\
\text { Exhala el aire que ha inspirado para respirar } \\
\text { Chillaría } \\
\text { Tiene células }\end{array}$ \\
\hline ÁRBOL & $\begin{array}{l}\text { Quitarle alimento } \longrightarrow \\
\text { Comprobar falta de nutrientes } \\
\text { y ausencia de luz } \longrightarrow \\
\text { Medir cada cierto tiempo } \\
\text { Plantar una semilla } \\
\text { Examinar una muestra de tejido } \\
\text { al microscopio }\end{array}$ & $\begin{array}{l}\text { Se muere } \\
\text { Se marchita } \\
\text { Crece } \\
\text { Se reproduce }\end{array}$ \\
\hline VACA & $\begin{array}{l}\text { Privarla de alimento } \longrightarrow \\
\text { Privarla de oxígeno } \longrightarrow \\
\text { Amenazarla con un palo } \longrightarrow \\
\text { Comprobar que se reproduce } \longrightarrow\end{array}$ & $\begin{array}{l}\text { Se muere } \\
\text { Se muere } \\
\text { Escaparía }\end{array}$ \\
\hline CRISTAL & $\begin{array}{l}\text { Aislarlo de su medio } \longrightarrow \\
\text { Cortar un trozo de un brazo } \longrightarrow \\
\text { Análisis y ver } \longrightarrow \\
\text { Tocarlo } \longrightarrow\end{array}$ & $\begin{array}{l}\text { No sobrevive } \\
\text { Vuelve a crecer } \\
\text { Si está constituido por células } \\
\text { Ver cómo reacciona }\end{array}$ \\
\hline
\end{tabular}

\section{Marco conceptual utilizado}

Los estudiantes, a la hora de emitir sus respuestas, se apoyaron fundamentalmente en características fisiológicas más que en criterios estructurales. Se aprecian, no obstante, diferencias entre ambos grupos de alumnos. Las respuestas funcionales son superiores en magisterio $(89,7 \%$ del total) que en biológicas $(75,2 \%)$. Por el contrario, en este último colectivo, se observa un incremento de las respuestas estructurales que, aun siendo minoritarias, ascienden al $24,8 \%$, mientras que en magisterio se sitúan en el 10,3\%. A continuación y con el fin de clarificar la exposición de los resultados, trataremos, en primer lugar, las respuestas fisiológicas y, en segundo término, las estructurales.

\section{Respuestas basadas en criterios fisiológicos}

En esta categoría, las funciones vitales más indicadas por los alumnos fueron las siguientes: alimentación/ nutrición, respiración, movimiento, crecimiento, respuesta a estímulos y reproducción. Existen, sin embargo, diferencias tanto entre los dos grupos de alumnos como entre los cuatro ejemplos propuestos. Así, los porcentajes de respuestas correspondientes a las distintas funciones y ejemplares (Fig. 1) muestran que:

- La alimentación/nutrición es la característica más señalada en los distintos ejemplos a excepción de «árbol» en biológicas. Concretamente, el porcentaje de alumnos de magisterio que han utilizado este criterio oscila entre el 90,2\% correspondiente a «vaca» y el 58,1\%, a «cristal», destacando asimismo el 70,7\% relativo al «árbol». En biológicas, los valores se encuentran entre el $69 \%$ para «niño» y el 39,5\% para «árbol».

- La respiración es utilizada como soporte conceptual por más de la mitad de los alumnos de los dos colectivos en el ejemplar «niño» así como por los de magisterio en 
Figura 1

Porcentaje de alumnos que utilizaron criterios fisiológicos en la diferenciación de ser vivo.

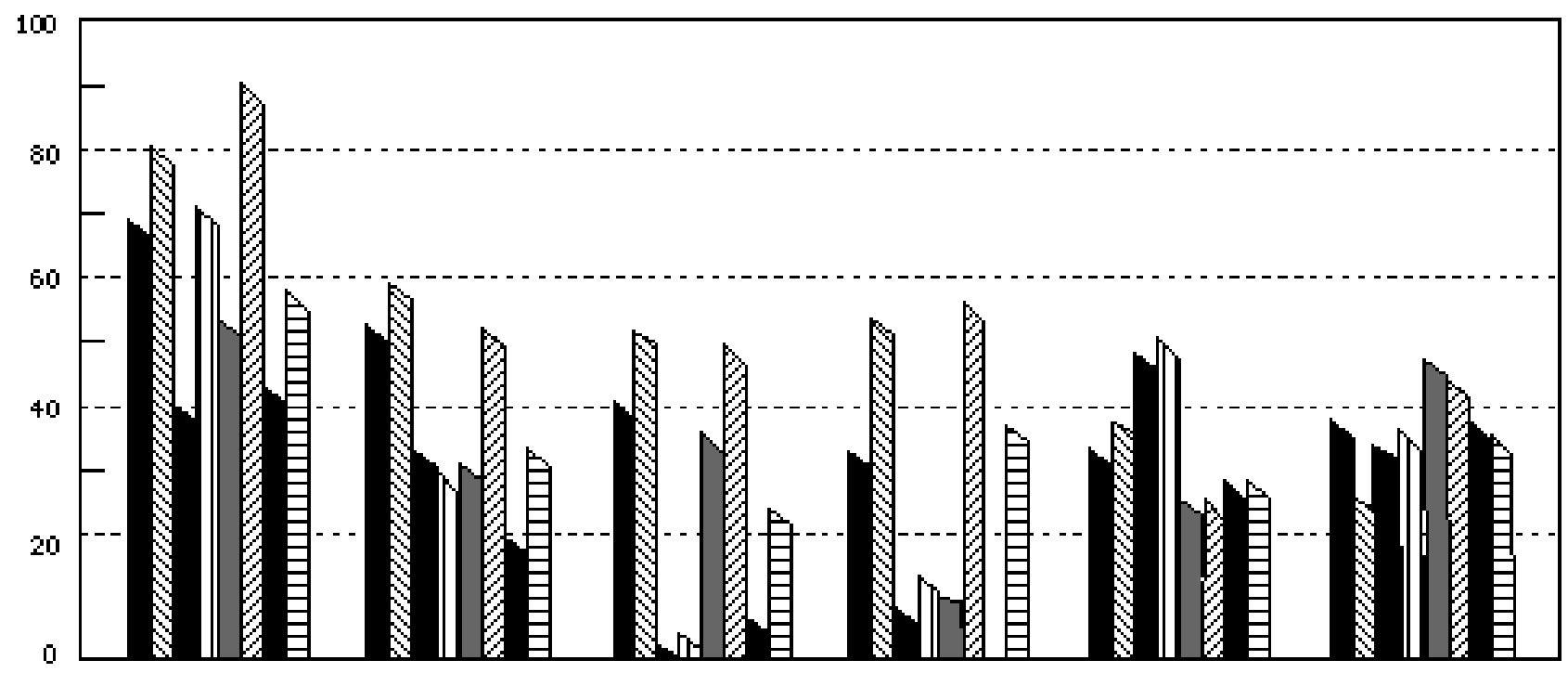

\begin{tabular}{|c|c|c|c|c|c|c|}
\hline & 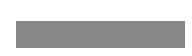 & 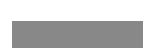 & 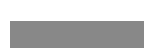 & 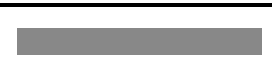 & 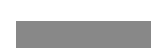 & $\bar{D}$ \\
\hline 口 & $\square$ & $\square$ & $\square$ & $\square$ & $\square$ & $\square$ \\
\hline 四 & $\square$ & $\square$ & $\square$ & $\square$ & $\square$ & $\square$ \\
\hline a & $\square$ & $\square$ & $\square$ & $\square$ & $\square$ & $\square$ \\
\hline 口 & $\square$ & $\square$ & $\square$ & $\square$ & $\square$ & $\square$ \\
\hline 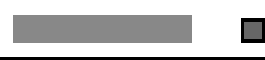 & $\square$ & $\square$ & $\square$ & $\square$ & $\square$ & $\square$ \\
\hline 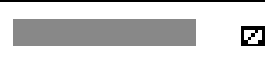 & $\square$ & 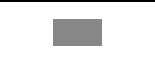 & $\square$ & $\square$ & 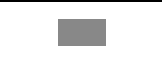 & $\square$ \\
\hline n & $\square$ & $\square$ & $\square$ & $\square$ & $\square$ & $\square$ \\
\hline 日 & $\mathbf{a}$ & 口 & $\square$ & $\square$ & $\square$ & $\square$ \\
\hline
\end{tabular}

«vaca». Estos porcentajes descienden en los dos ejemplares restantes y en especial en el correspondiente a «cristal» en el grupo de biológicas (19\%).

- Tanto los alumnos de magisterio como los de biológicas han tenido en cuenta el movimiento en mayor medida en «niño» (el 52,2\% y 40,5\%, respectivamente) y «vaca» $(49,9 \%$ y $35,7 \%$ respectivamente) que en «cristal» (no superó el 25\%) y «árbol» (no superó el 5\%).

- En relación con la respuesta a estímulos se observa la misma pauta anterior, siendo menos señalada en «árbol» $\mathrm{y}$ «cristal». En concreto, los porcentajes correspondientes a los alumnos de magisterio están comprendidos entre el 56,5\% («vaca») y el 13,6\% («árbol») y los de biológicas entre el 33,3\% para «niño» y el $0 \%$ para «cristal».
- Se detecta una mayor concordancia en el porcentaje de alumnos de ambos colectivos que se han apoyado en el crecimiento para cada ejemplar. Los valores más altos recaen en «árbol», al haber sido señalado por alrededor del $50 \%$ de los alumnos, y los inferiores, que giran en torno al $26 \%$ en los dos grupos, se corresponden con el ejemplar «vaca».

- Los porcentajes relativos a la reproducción son relativamente similares en todos los ejemplares y en ambos colectivos. Se aprecia, no obstante, un incremento para «vaca», ejemplar en el que dicha función es mencionada aproximadamente por el $45 \%$ de los alumnos de cada grupo.

Además de las funciones vitales señaladas hasta el momento y que en mayor o menor medida afectan a todos 
Figura 2

Porcentaje de alumnos que utilizaron criterios estructurales en la diferenciación de ser vivo.

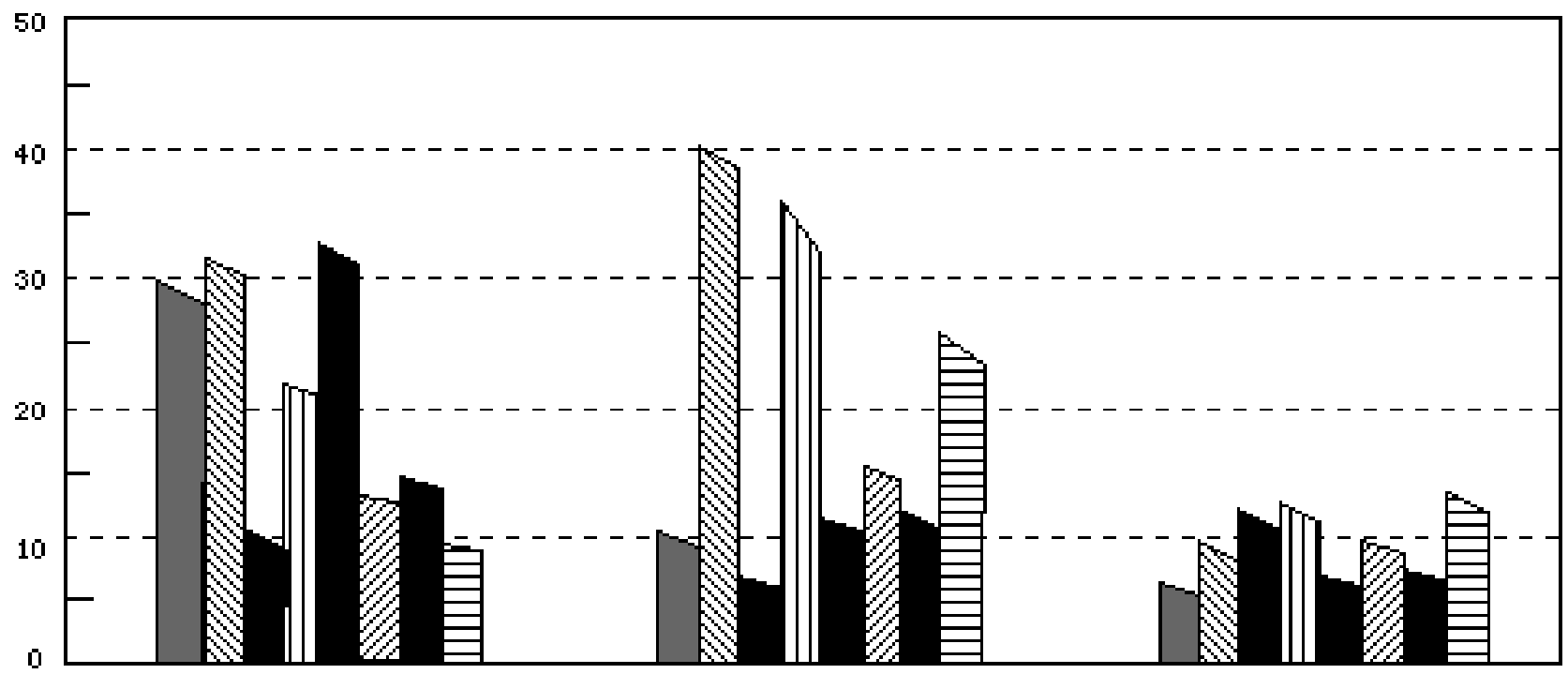

\begin{tabular}{|c|c|c|c|c|}
\hline & & 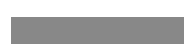 & 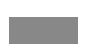 & 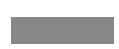 \\
\hline & 口 & $\square$ & $\square$ & $\square$ \\
\hline - & $\Delta$ & $\square$ & $\square$ & $\square$ \\
\hline & घ & $\square$ & $\square$ & $\square$ \\
\hline & $\mathbb{\square}$ & $\square$ & $\square$ & $\square$ \\
\hline 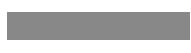 & घ & $\square$ & $\square$ & $\square$ \\
\hline 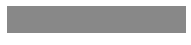 & Q & $\square$ & $\square$ & $\square$ \\
\hline & a & $\square$ & $\square$ & $\square$ \\
\hline 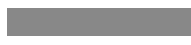 & 日 & $\square$ & $\square$ & $\square$ \\
\hline
\end{tabular}

los ejemplares, los alumnos han propuesto otras más específicas, que atañen a ejemplares concretos. Entre ellas destacan: la relación con otros seres vivos, en los dos ejemplos de animales (alrededor del 25\%); la audición, lenguaje, sentimientos y pensamiento apuntados por un $30 \%$ de los alumnos de magisterio en el caso concreto del «niño»; la adaptación al medio en «cristal» (35,2\% en magisterio frente al $11,9 \%$ de biológicas) y la fotosíntesis en «árbol», a la que han hecho referencia el $25,6 \%$ de los alumnos de biológicas y el 34,8\% de magisterio.

El análisis cualitativo de las respuestas fisiológicas muestra que los alumnos basan sus observaciones y experiencias fundamentalmente en aspectos macroscópicos y perceptibles -alimentación, respiración, etc.- siendo muy minoritarias las propuestas fundamentadas en la fisiología celular-absorción de nutrientes, metabolismo, división celular-, tisular-contracción muscular, transporte, etc.o desde el punto de vista de órganos - procesos implicados en la nutrición, por ejemplo-. Así, la respiración se asocia exclusivamente con el intercambio de gases tal y como se desprende de respuestas representativas como puede ser «observar su respiración». Además, el análisis de las propuestas sugeridas por los alumnos también revela que tras una terminología científica se oculta un importante número de concepciones y confusiones conceptuales especialmente en el grupo de magisterio. Es 
así muy frecuente que utilicen alimentación y nutrición como sinónimos y que mantengan concepciones científicamente incorrectas acerca de la fotosíntesis. Con el fin de ilustrar los aspectos apuntados citaremos literalmente algunas respuestas representativas tales como: «observar si lleva a cabo el proceso de nutrición; puedo observar cómo se nutre: qué come, cuándo, le gusta o no...; el árbol se alimenta de sustancias del suelo; este ejemplar es un ser vivo porque si tú a esta planta no la alimentas, no la riegas, se muere; compruebo que la falta de alimento y la ausencia de luz producen que la planta se marchite...».

La comprobación de otras características fisiológicas, como movimiento, crecimiento o reproducción, está asimismo referida a sus aspectos visibles, si bien pueden involucrar tanto al organismo $\mathrm{u}$ objeto inerte en su totalidad (sobre todo en movimiento y crecimiento) como a partes concretas de los mismos. En este sentido falta toda referencia al movimiento celular así como a la división mitótica o a la formación de gametos. Ejemplos de respuestas literales muy generalizadas, pueden ser: «demostrar que crece, comprobar que se mueve, si cortamos una rama vuelve a crecer, ver si el ejemplar se desplaza o si mueve alguna parte de su cuerpo, observar si se reproduce...».

En relación con la respuesta a estímulos se aprecian afirmaciones que la mencionaban como una característica general de los seres vivos y afirmaciones restringidas a algún ejemplar al cual se aplicaba un estímulo específico. En este último caso, los alumnos se ciñeron a los estímulos físicos con alta predominancia de los mecánicos (pinchar, tocar...) aunque ocasionalmente también aludieron a los luminosos y térmicos (fototropismo, respuestas a temperaturas altas o demasiado bajas). Algunas respuestas textuales en este sentido son las siguientes: «los seres vivos responden a estímulos externos, si toco un brazo del ejemplar se retrae, lo meto en una habitación muy caliente y observo su respuesta, lo pincho y observo su reacción».

\section{Respuestas basadas en criterios estructurales}

Estas respuestas fueron minoritarias. Los estudiantes hicieron referencia a sistemas/órganos y/o tejidos, a la célula y a la composición química como características diferenciadoras de seres vivos y materia inerte, apreciándose diferencias en cuanto al grupo de alumnos y a los distintos ejemplares (Fig. 2). Assí:

- La presencia de sistemas y/o de órganos y tejidos es contemplada en «niño» por alrededor de un $30 \%$ de los estudiantes de ambos colectivos. En el ejemplar «vaca», se mantiene aproximadamente en los límites anteriores, en magisterio, con un $32,1 \%$, y disminuye hasta el 14,3\% en biológicas. Estos porcentajes descienden en ambos grupos de alumnos en referencia a «cristal» y en el grupo de magisterio en referencia a «árbol» $(9,8 \%)$.

- La constitución celular ha sido señalada por un reducido número de estudiantes de magisterio (los valores obtenidos oscilan entre el $6 \%$ correspondiente al ejemplar «árbol»y el 11,7\% a «cristal»). Las referencias celulares se incrementan de manera apreciable en biológicas puesto que el 40,5\% señaló su presencia en «niño» y el 34,9\% en «árbol». Los porcentajes son, sin embargo, inferiores en «cristal» $(23,9 \%)$ y en «vaca» $(16,7 \%)$.

- El número de alumnos que hizo alusión a la composición química específica de los seres vivos (proteínas, ácidos nucleicos...) es inferior al $12 \%$ en ambos colectivos para todos los ejemplos.

Debemos destacar que las respuestas de los estudiantes respecto a este tipo de estructuras suelen ser, por lo general, ambiguas, imprecisas e indirectas. Abundan contestaciones como: «análisis interno observando órganos y sistemas; observo como está constituido su cuerpo, analizar una muestra de tejido; compararlo con seres vivos semejantes; analizar sus raíces...».

\section{DISCUSIÓN}

Uno de los primeros resultados obtenidos en este trabajo, y que confirma nuestra hipótesis de partida, revela la dificultad que presentan los alumnos consultados para proponer experimentos. Sus respuestas así lo indican puesto que en su gran mayoría están basadas en descripciones u observaciones cualitativas directas que, si bien son válidas para identificar órganos, sistemas de órganos y funciones características de gran número de seres vivos, no siempre son extrapolables a otras formas vivas de menor nivel de organización. Además, las pruebas propuestas son pobres e imprecisas, observándose en ambos grupos la ausencia de respuestas que reflejen hipótesis explícitas, técnicas manipulativas, como la realización de cortes, toma de datos, interpretación de resultados y conclusiones. Así, el hecho de que la mayoría de los ensayos estén formulados en términos de acción-consecuencia (Tabla I), o lo que es lo mismo de causa-efecto, hace que se asemejen a los sugeridos en un estudio realizado con estudiantes de 13 años de edad (Osaki y Samiroden, 1990).

Aunque los alumnos de magisterio proponen más acciones a realizar sobre los distintos ejemplares, no indican por lo general el resultado de tal acción, aspecto que sí señalan mayoritariamente los de biológicas. Esta diferencia entre ambos colectivos puede deberse a los mayores conocimientos conceptuales de los estudiantes de biología y no a su mayor familiarización con la metodología científica.

Por otra parte, el aumento del número de respuestas de contrastación de hipótesis -cambio de hábitat, ensayos para observar si existe reproducción asexual o regeneración de una parte, etc.- detectado en «cristal» respecto a otros ejemplares podría obedecer al desconcierto de los alumnos ante un objeto no familiar. En este caso, los aspectos perceptibles no resultan suficientemente evidentes, requiriendo la realización de ensayos. De hecho, como hemos visto, tanto en este ejemplar como en 
«árbol» se observa un ligero aumento de las propuestas «experimentales».

Posiblemente, la dificultad para utilizar determinados procedimientos científicos puesta en evidencia en otros estudios (Brumby, 1982; Osaki y Samiroden, 1990) haya que atribuirla a la enseñanza recibida. En este sentido, cabe señalar que los libros de texto habitualmente utilizados por los profesores de BUP y COU no suelen incluir procedimientos científicos básicos (Tamir y García, 1992) y que, por su parte, las prácticas habituales tampoco proporcionan a los alumnos la oportunidad de familiarizarse con aspectos elementales de la metodología científica tales como la emisión de hipótesis, la observación rigurosa, el diseño de experimentos, el análisis crítico de resultados... (Gil y Payá, 1988; García et al., 1995).

Con relación a los atributos vitales que han tenido en cuenta los alumnos a la hora de diferenciar ser vivo de materia inerte, objetivo fundamental de este trabajo, debemos destacar la preponderancia de las respuestas basadas en criterios fisiológicos frente a las fundamentadas en aspectos estructurales y la variación de tales respuestas en función del ejemplar y del colectivo consultado.

La alimentación/nutrición es la característica vital más señalada por ambos grupos de alumnos, en especial por los de magisterio, en la mayoría de los ejemplares, lo que contrasta con los resultados obtenidos, entre otros, por Lucas y otros (1979) y por Brumby (1982). De acuerdo con estos autores, el movimiento sería el criterio más utilizado por alumnos de niveles educativos obligatorios y universitarios para averiguar si un objeto no familiar es o no un ser vivo. En nuestro estudio, por el contrario, el movimiento presenta una menor relevancia incluso en el caso de ejemplares conocidos y claramente móviles como «niño» y «vaca». El acusado descenso en la aplicación de dicho criterio en «cristal»-objeto no familiarpodría deberse al aspecto que ofrecía el mineral elegido, que indujo a una buena parte del alumnado a suponer que se trataba de una colonia de corales. Este descenso, unido a las escasas referencias al movimiento en «árbol», es indicativo de que los alumnos tienen exclusivamente en cuenta el movimiento directamente observable.

Los alumnos, y en especial los de magisterio, restringen el proceso nutritivo al ámbito macroscópico utilizándolo como sinónimo de alimentación y refiriéndose muy escasamente al metabolismo celular. Se podría pensar que las bajas referencias a los procesos metabólicos son debidas a la dificultad que tienen los alumnos para proponer experimentos en el ámbito molecular. Sin embargo, debemos tener en cuenta que tampoco mencionan este nivel en sus descripciones. Por ello nos inclinamos a pensar que es su visión del proceso nutritivo lo que les ha permitido proponer observaciones e incluso experimentos muy simples con el consiguiente incremento de esta categoría de respuesta. Asimismo, al confundir alimentación con nutrición, consideran que las plantas también se alimentan, idea que concuerda con los resultados obtenidos en otras investigaciones (véase, por ejemplo, la revisión hecha por Wood-Robinson, 1991). Tal concepción explicaría el hecho de que en el ejemplar «árbol» la alimentación/nutrición haya sido más mencionada que el proceso fotosintético. El hecho de que en el grupo de magisterio se haya detectado una mayor presencia de las concepciones anteriormente citadas responde, probablemente, al menor número de cursos de biología seguidos por estos alumnos, ya que aproximadamente la tercera parte de ellos no han optado por la asignatura de ciencias naturales en $3^{\circ}$ de BUP o por la de biología en COU.

La utilización de otras funciones fisiológicas como respiración, respuesta a estímulos y crecimiento se muestra asimismo dependiente del ejemplar considerado. Conviene destacar en este sentido el descenso generalizado de las referencias a la respiración y a la respuesta a estímulos en los ejemplares «árbol» y «cristal», mientras que el crecimiento, además de ser una de las características más atribuidas al «árbol», también ha sido reconocido en mayor medida en este ejemplar que en los demás. Este énfasis en la atribución de crecimiento al «árbol», probablemente debido a que es una de sus características más perceptibles y vivenciales, también ha sido puesto de manifiesto por Stavy y Wax (1989), al detectar que esta característica es universalmente atribuida a las plantas por estudiantes de todas las edades, incluso por aquéllos que ni siquiera las consideran seres vivos. En lo que respecta a la respiración y al igual que ocurría con la nutrición, los alumnos presentan una importante limitación conceptual al no asociar ambos procesos y al referirse mayoritariamente a la respiración en términos de intercambio de gases. Es decir, los estudiantes no la identifican como un proceso celular productor de energía, idea ya evidenciada en otros estudios (Gayford, 1986; Haslam y Treagust, 1987; Anderson et al., 1990; Banet y Núñez, 1990; Songer y Mintzes, 1994). Por otra parte, es habitual que estudiantes de distintos niveles educativos tengan escasamente en cuenta la respiración en vegetales y mantengan distintas concepciones sobre la misma (Arnold y Simpson, 1980; Simpson y Arnold, 1982a; Bell, 1985), lo que explicaría la disminución de las referencias al proceso en el ejemplar «árbol».

Asimismo, las respuestas relativas a estímulos presentan una especial relevancia. Las aportaciones de otros estudios acerca de la atribución de esta característica a los seres vivos se refieren fundamentalmente a trabajos realizados en niveles educativos inferiores en los que dicha atribución es realmente muy minoritaria (Osaki y Samiroden, 1990). En este sentido, sorprende observar que este criterio haya sido relativamente considerado por los estudiantes de magisterio mientras que los de biológicas no lo han tenido en cuenta o lo han considerado escasamente. Es posible que dicha diferencia radique, siguiendo la argumentación utilizada por Dreyfus y Jungwirth (1989), en que los alumnos de magisterio aplican, en mayor medida que los de biológicas, su conocimiento personal a las situaciones escolares . Así, el hecho de que mencionen respuestas a estímulos físicos en los ejemplares «niño» y «vaca», responde seguramente a las propias experiencias o a las percibidas en otros organismos de su entorno. 
El análisis hasta el momento realizado nos permite apreciar que los estudiantes no señalan los procesos metabólicos, reduciendo de este modo la fisiología a sus niveles macroscópicos o perceptibles. Además, los elevados porcentajes de respuestas relativos a funciones vitales indican que los alumnos consideran en todo momento que los ejemplares están vivos, aunque sí se han referido a la muerte como algo inherente a la vida.

Por otra parte, todo parece indicar que la focalización en los aspectos más perceptibles ha influido en la menor utilización de criterios estructurales microscópicos (célula, DNA), esenciales y suficientes para diferenciar ser vivo de materia inerte.

En este sentido, los alumnos de biológicas han reconocido la presencia de las células en mayor medida que los de magisterio. No obstante y dada la especialidad elegida por este primer colectivo, sorprende constatar las escasas respuestas referidas a la estructura celular de los seres vivos. Creemos que tal escasez es indicativa no de la falta de conocimientos de los alumnos sino de la dificultad que tienen para aplicarlos en contextos no académicos. Además, la variabilidad mostrada en sus respuestas en relación con los distintos objetos presentados resulta sorprendente, ya que admiten en mayor medida la estructura celular de «niño» que de «vaca» a pesar de las semejanzas existentes entre ambos. También cabría esperar que, en «cristal»-nuestro objeto no familiar-, los alumnos se apoyasen mayoritariamente en la estructura celular con el fin de conocer si ese ejemplo era, o no, un ser vivo, tal y como se podría desprender de los resultados obtenidos por Brumby (1982), ya mencionados anteriormente, en una tarea similar llevada a cabo con estudiantes universitarios. Con relación al colectivo de magisterio es preciso destacar que las respuestas celulares son especialmente minoritarias, muy poco acordes con la enseñanza recibida y más en consonancia con las halladas por Lucas y otros (1987) en noveno grado (15 años). Ello confirma la dificultad que poseen los estudiantes para reconocer la célula como unidad de vida (Caballer y Jiménez, 1992; Dreyfus y Jungwirth, 1988; 1989).

Asimismo, resulta llamativo comprobar que los estudiantes de ambos grupos omiten o apenas apuntan la presencia de moléculas concretas como DNA, proteínas, etc. Este hecho, también señalado por otros autores (Lucas et al., 1979; Brumby, 1982), es concordante con las escasísimas menciones a los procesos metabólicos celulares.

Tampoco las estructuras con mayor nivel de organización, incluso macroscópicas, como sistemas y órganos, han sido muy consideradas por los estudiantes, aunque el colectivo de magisterio las ha tenido más en cuenta que la constitución celular. Se constata así la existencia de un notable desequilibrio entre las referencias fisiológicas y las estructurales, que denotan la ausencia de las oportunas relaciones entre estructura y función. Probablemente, tal desequilibrio haya que atribuirlo a la mayor facilidad que tienen los estudiantes para fijarse en aspectos dinámicos, perceptibles (ingestión de alimentos, intercambio de gases, etc.), minimizando lo estático, lo que atañe a cambios no observables aunque se trate de estructuras macroscópicas.

Si bien las diferencias observadas entre los grupos de alumnos consultados, anteriormente comentadas, pueden ser debidas, como también se ha sugerido, al mayor o menor conocimiento conceptual que éstos poseen, éste no es suficiente para explicar las variaciones apreciadas en las diversas respuestas dadas por cada uno de los grupos para los distintos ejemplares. En este sentido, se puede decir que el grado de consistencia mostrado por los alumnos en sus respuestas es bajo, ya que sólo una minoría emite la misma para los cuatro ejemplos propuestos. Otros autores también han detectado la inconsistencia de los alumnos en la generalización del concepto de vida (Tamir et al., 1981; Carey, 1985; Stavy y Wax, 1989; Strommen, 1995). Una de las causas de tal inconsistencia se debería a que el pensamiento de los alumnos, al igual que el pensamiento humano, se rige más por criterios funcionales que por criterios formales (Manuel y Grau, 1996), por lo que no consideran la necesidad de ser coherentes en su razonamiento, aspecto que sí es esencial para la comunidad científica (Hewson, 1985). Otra posible causa sería que los alumnos centran su atención en aspectos particulares y directamente observables más que en la búsqueda de criterios generales aplicables a distintos contextos (Viennot, 1985; Engel y Driver, 1986; Hewson, 1990).

En resumen, los resultados obtenidos en este trabajo permiten apreciar que, en general, los estudiantes consultados no usan los criterios básicos universales ya citados (célula, DNA) característicos del concepto ser vivo. Por el contrario, a pesar de la enseñanza recibida, su atención se centra fundamentalmente en aspectos perceptibles o macroscópicos, lo que pone de manifiesto la utilización de un pensamiento cotidiano. Además, no debemos olvidar que la consideración de características vitales desde el punto de vista microscópico o submicroscópico constituye un obstáculo epistemológico difícil de superar, condicionando, por consiguiente, las respuestas del alumnado. En este sentido, la enseñanza ha de procurar que los alumnos superen los problemas que supone la relación entre lo macroscópico y lo microscópico. En esta línea Dreyfus y Jungwhirt (1988) consideran que el estudio de la célula como unidad viva es fundamental para comprender el funcionamiento de los organismos pluricelulares. En nuestra opinión, este tratamiento de la célula como unidad viviente constituye un paso importante para que el concepto de vida y sus manifestaciones observables avancen hacia una perspectiva universal y microscópica. 


\section{REFERENCIAS BIBLIOGRÁFICAS}

ANDERSON, C.W., SHELDON, T.H. y DUBAY, J. (1990). The effects of instruction on college nonmajors' conceptions of respiration and photosynthesis. Journal of Research in Science Teaching, 27(8), pp. 761-776.

ARNOLD, B. y SIMPSON, M. (1980). An investigation of the Development of the concept Photosynthesis to SCE «O» Grade.Aberdeen: Aberdeen College of Education, Aberdeen, Reino Unido.

BANET, E. y NÚÑEZ, F. (1990). Esquemas conceptuales de los alumnos sobre la respiración. Enseñanza de las Ciencias, 8(2), pp. 105-110.

BELL, B. y FREYBERG, P. (1991). El lenguaje en la clase de ciencias, pp. 56-73, en Osborne, R. y Freyberg, P., El aprendizaje de las Ciencias. Implicaciones de la ciencia de los alumnos. Madrid: Narcea.

BELL, B. (1985). Students' ideas about plant nutrition: What are they? Journal of Biological Education, 19(3), pp. 213-218.

BRUMBY, M. (1982). Student's perceptions of the concept of life. Science Education, 66(4), pp. 613-622.

BUNGE, M. (1969). La investigación científica. Barcelona: Ariel.

CABALLER, M. J. y JIMÉNEZ, I. (1992). Las ideas de los alumnos y alumnas acerca de la estructura celular de los seres vivos. Enseñanza de las Ciencias, 10(2), pp. 172-180.

CAREY,S. (1985). Conceptual change in childhood. Cambridge. MA: MIT Press.

DREYFUS, A. y JUNGWIRTH, E. (1988). The cell concept of 10 th graders: curricular expectations and reality. International Journal of Science Education, 10(2), pp. 221-229.

DREYFUS, A. y JUNGWIRTH, E. (1989). The pupil and the living cell: a taxonomy of disfunctional ideas about an abstract idea. Journal of Biological Education, 23(1), pp. 49-55.

ENGEL, E. y DRIVER, R. (1986). A study of consistency in the use of students' conceptual frameworks across different task contexts. Science Education, 70(4), pp. 473-496.

GAYFORD, C.G. (1986). Some aspects of the problems of teaching about energy in school biology. European Journal of Science Education. 8(4), pp. 443-450.

GARCÍA, S., MARTÍNEZ, M.C. y MONDELO, M. (1995). El trabajo práctico. Una intervención para la formación de profesores. Enseñanza de las Ciencias, 13(2), pp. 203-209.

GIL, D. y PAYÁ, J. (1988). Los trabajos prácticos de física y química y la metodología científica. Revista de Enseñanza de la Física, 2(2), pp. 73-79.

HASLAM, F. y TREAGUST, D.F. (1987). Diagnosing secondary students' misconceptions of photosynthesis and respiration in plants using a two-tier multiple choice instrument. Journal of Biological Education, 21(3), pp. 203-211.

HEWSON, P.W. (1985). Epistemological commitments in the learning of science: Examples for dynamics. European Journal of Science Education, 7(2), pp. 163-172.

HEWSON, P.W. (1990). La enseñanza de «fuerza y movimiento» como cambio conceptual. Enseñanza de las Ciencias, 8(2), pp. 157-171.
JOUVE, N. (1996). Avances en genética y su utilización en la enseñanza no universitaria. Alambique, 10, pp. 69-78.

LOOFT, W.R. (1974). Animistic thought in children: Understanding of «living» across its associated attributes. Journal of Genetic Psychology, 124, pp. 235-240.

LUCAS, A.M., LINKE, P.P. y SEDWICK, J. (1979). Schoolchildren's criteria for «alive»: a content analysis approach. Journal of Psychology, 103, pp. 103-112.

MANUEL, J. de y GRAU, R. (1996). Concepciones y dificultades comunes en la construcción del pensamiento biológico. Alambique 7, pp. 53-63.

MONDELO, M., GARCÍA, S. y MARTÍNEZ, C. (1994). Materia inerte - materia viva ¿Tienen ambas constitución atómica? Enseñanza de las Ciencias, 12(2), pp. 226-233.

OSAKI, K. y SAMIRODEN, W.D. (1990). Children's conceptions of 'living' and 'dead'. Journal of Biological Education, 24(3), pp. 199-207.

PIAGET, J. (1929). The Child's conception of the World. Nueva York: Harcourt, Brace.

RUSSELL, R.W. y DENNIS, W. (1939). Studies in animism.I. A standardized procedure for the investigation of animism. Journal of Genetic Psychology, 55, pp. 389-400.

SIMPSON, M. y ARNOLD, B. (1982). The inappropriate use of subsumers in biology learning. European Journal of Science Education, 4(2), pp. 173-182.

SMEETS, P.M. (1973). The animism controversy revisited: A probability analysis. Journal of Genetic Psychology, 123, pp. 219-225.

SONGER, C.J. y MINTZES, J.J. (1994). Understanding cellular respiration: An Analysis of conceptual change in college Biology. Journal of Research in Science Teaching, 31(1), pp. 621-637.

STAVY, R. y WAX, N. (1989). Children's conceptions of plants as living things. Human Development, 32, pp. 88-94.

STROMMEN, E. (1995). How are living things alike and different? First graders' knowledge of basic life science concepts. Journal of Biological Education, 29(4), pp. 286-292.

TAMIR,P., GAL-CHOPPIN, R. y NUSSINOVITZ, R. (1981) How do intermediate and junior high school students conceptualize living and non living? Journal of Research in Science Teaching, 18(3), pp. 249-254.

TAMIR, P. y GARCÍA, M.P. (1992). Características de los ejercicios de prácticas de la laboratorio incluidos en los libros de texto de ciencias usados en Cataluña. Enseñanza de las Ciencias, 10(1), pp. 3-12.

VIENNOT, L. (1985). Analising students' reasoning in Science: A pragmatic view of theoretical problems. European Journal of Science Education, 7(2), pp. 151-162.

WOOD-ROBINSON, C. (1991). Young people's ideas about plants. Studies in Science Education, 19, pp. 119-135.

WOOD-ROBINSON, C. (1994). Young people's ideas about inheritance and evolution. Studies in Science Education, 24, pp. 29-47.

[Artículo recibido en febrero de 1997 y aceptado en enero de 1998.] 